\title{
A narrative review of human epidermal growth factor receptor 2 (HER2)-targeted antibody-drug conjugates in the treatment of breast cancer
}

\author{
Qun Li, Ye Guo, Fengjuan Lin, Lingjun Li, Xiaoxiao Ge, Wei Zhao, Jin Li^^ \\ Department of Medical Oncology, Tongji University Shanghai East Hospital, Shanghai, China \\ Contributions: (I) Conception and design: Q Li, J Li; (II) Administrative support: Y Guo; (III) Provision of study materials or patients: L Li, X Ge; (IV) \\ Collection and assembly of data: Q Li, W Zhao; (V) Data analysis and interpretation: Q Li; (VI) Manuscript writing: All authors; (VII) Final approval \\ of manuscript: All authors. \\ Correspondence to: Jin Li. Department of Medical Oncology, Tongji University Shanghai East Hospital, No. 1800 Yuntai Road, Pudong New Area, \\ Shanghai, China. Email: lijin@csco.org.cn.
}

Objective: The aim of this study was to summarize the human epidermal growth factor receptor 2
(HER2)-targeted antibody-drug conjugates (ADCs) for the treatment of breast cancer.
Background: ADCs are a new and promising kind of antitumor targeted drug valued for their efficient
drug delivery and controllable side effects. Among the ADC targets, HER2 is one of the most studied.
It is the target which can yield the most benefit, as breast cancer patients expressing HER2 usually have
aggressive disease and a poor prognosis. Both the approved and clinical trials ongoing for HER2-targeted
ADCs have shown their efficacy for breast cancer, especially in trastuzumab treatment-failed cases.
Methods: We searched relevant studies published in English in the PubMed, ClinicalTrials.gov, google.
com, and in Chinese in NMPA website up to Sep 9, 2021, using the search terms "HER2 ADC", "breast
cancer", "antibody drug conjugate (ADC)", "clinical trial". HER2-targeted ADCs have changed the
treatment of HER2-positive breast cancer considerably; however, some issues still need to be resolved. For
one, it is necessary to find cytotoxic drugs and linkers with better efficacy and lower toxicity. For another,
ADC as a single drug has already shown potent effect, but its combination with other treatments, such as
programmed cell death protein 1/programmed death-ligand 1 (PD-1/PD-L1) antibody, may produce even
greater results.

Conclusions: The approved HER2-targeted ADCs have significantly benefited breast cancer patients, while those still being investigated will likely prove to offer even further value.

Keywords: Human epidermal growth factor receptor 2 (HER2); antibody-drug conjugates (ADCs); HER2targeted ADCs; breast cancer

Received: 11 October 2021; Accepted: 30 October 2021; Published: 31 October 2021.

doi: 10.21037/tbcr-21-33

View this article at: https://dx.doi.org/10.21037/tbcr-21-33

\section{Introduction}

Traditional chemotherapy can eliminate tumors but also cause systemic side effects, and the curative effect and side effects must be balanced, which limits the efficacy of the related drugs (1). Antibody-drug conjugates (ADCs) can selectively deliver the cytotoxic drug that is conjugated with an antibody to the tumor by the specific binding of the antibody (2). An ADC consists of 3 parts: a monoclonal antibody, a cytotoxic drug (also known as the "payload"),

^ ORCID: 0000-0002-3172-8913. 


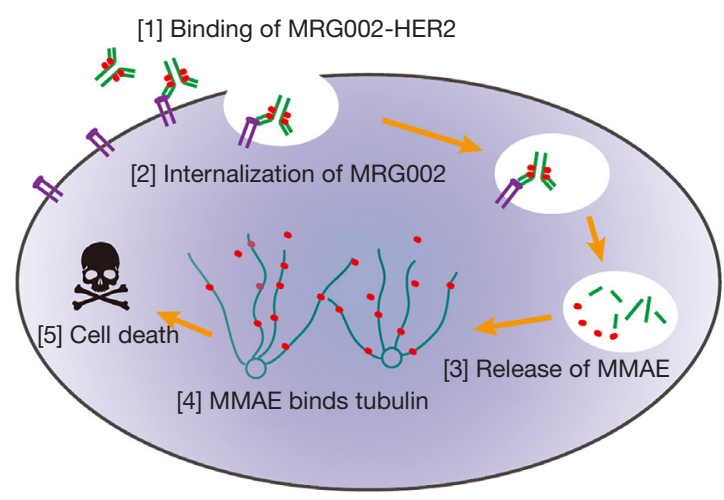

Figure 1 Antitumor mechanism of MRG002. HER2, human epidermal growth factor receptor 2; MMAE, monomethyl auristatin E.

and a linker.

When ADCs enter blood system, the antibody can recognize the target and then bind with the antigen on the cell. ADCs enter the tumor cells via the endocytosis of cells. Once the ADC is in the cell, the linker between the antibody and cytotoxic is cleaved by the lysosome and the payload is released intracellularly (3). Because of this mechanism, most of the early clinical application of ADCs has mainly involved treating hematological tumors. However, follow-up studies have confirmed that ADC also has a good effect in solid tumors. Despite the concept being proven in 1975, the first ADC drug was not approved by the US Food and Drug Administration (FDA) until 2000, and now more than 10 ADCs have been approved with more being under current clinical evaluation (4).

Breast cancer is the most common malignant tumor in women (5). Among ADCs for breast cancer treatment, the most common one targets human epidermal growth factor receptor 2 (HER2), which is expressed in a variety of cancers, especially breast cancer (6). HER2-positive breast cancer accounts for about $20 \%$ of all breast cancer cases, is highly invasive, and prone to early recurrence and metastasis. Anti-HER2 therapy is the cornerstone of treatment for patients with HER2-positive breast cancer, with previous anti-HER2 treatments consisting mainly of monoclonal antibodies. Trastuzumab was the first monoclonal antibody approved for breast cancer and is considered to be one of the most significant advances in breast cancer treatment thus far. HER2-targeted ADCs may represent the next breakthrough in this area (7). In this review, we summarized the HER2-targeted ADCs for the treatment of breast cancer around the world and their investigation progress. Mechanisms of different HER2targeted ADCs are similar, with the main differences being the variety of linkers and payloads. The antitumor mechanism of MRG002, a typical new generation ADC with a cleavable linker and monomethyl auristatin $\mathrm{E}$ (MMAE) as the cytotoxic drug, is depicted in Figure 1. We present the following article in accordance with the Narrative Review reporting checklist (available at https:// dx.doi.org/10.21037/tbcr-21-33).

\section{Methods}

We searched relevant studies published in English in the PubMed, ClinicalTrials.gov, google.com, and in Chinese in NMPA website up to Sep 9, 2021, using the search terms "HER2 ADC", "breast cancer", "antibody drug conjugate (ADC)", "clinical trial".

\section{Approved HER2-targeted ADCs for breast cancer}

\section{Ado-trastuzumab emtansine}

T-DM1 is the first ADC approved for breast cancer. Its approved indication was for the treatment of advanced HER2-positive breast cancer, and it was subsequently approved for high-risk patients with early breast cancer with residual disease after neoadjuvant therapy. The antibody in T-DM1 is trastuzumab, and the cytotoxic drug is maytansine (DM1), with these 2 elements being connected by an uncleavable linker (8). As a natural product, DM1 is derived from the bark of the African shrub Maytenus ovatus, and it is an effective microtubule inhibitor. DM1 can inhibit cell division by suppressing the activity of the tubulin system, which ultimately leads to apoptosis. The antibody in T-DM1 is unmodified trastuzumab, which means that T-DM1 simultaneously exerts an antibodydependent cellular cytotoxicity (ADCC) effect and the signal suppression function of trastuzumab (9). On average, 3.5 DM1 molecules are linked to the antibody by the linker.

In the phase I studies, T-DM1 showed favorable safety in patients with HER2-positive metastatic breast cancer who had previously received a trastuzumab-containing regimen (10-12). The results of phase II clinical trials indicated the clinical benefit rate in patients treated with T-DM1 to be higher and the toxicity rate to be lower than those of the standard treatment regimen $(13,14)$. The result of phase III clinical studies EMILIA, TH3RESA, and MARIANNE showed that T-DM1 can benefit metastatic 
Table 1 Approved HER2-targeted ADCs for breast cancer

\begin{tabular}{llll}
\hline ADC & Linker & Cytotoxic compound & Indication \\
\hline $\begin{array}{l}\text { Ado-trastuzumab emtansine, } \\
\text { T-DM1, Kadcyla }\end{array}$ & Uncleavable & DM1 & HER2-positive early breast cancer with residual \\
& & & $\begin{array}{l}\text { invasive disease after neoadjuvant taxane and } \\
\text { trastuzumab-based treatment }\end{array}$ \\
$\begin{array}{l}\text { Trastuzumab deruxtecan, } \\
\text { Ds-8201a }\end{array}$ & Cleavable vc-linker & Deruxtecan & $\begin{array}{l}\text { Advanced HER2-positive breast cancer treated with at } \\
\text { least two prior lines of HER2-targeting therapy }\end{array}$ \\
\hline
\end{tabular}

HER2, human epidermal growth factor receptor 2; ADC, antibody-drug conjugate.

breast cancer patients $(15,16)$. In the TH3RESA study, the overall response rate (ORR) was $31.3 \%$ in the T-DM1 arm compared with only $8.6 \%$ in the physician's choice arm while the median progression-free survival (PFS) and overall survival (OS) were 6.2 and 3.3 months, respectively, and 22.7 and 15.8 months, respectively.

The KATHERINE study compared the efficacy of T-DM1 and trastuzumab. The results showed that the use of T-DM1 for intensive adjuvant therapy reduced the risk of recurrence or death of patients by $50 \%$ compared with that of the trastuzumab group, and the rate of invasive diseasefree survival (DFS) was lower than that of the trastuzumab group. Therefore, for patients with HER2-positive breast cancer who have undergone neoadjuvant treatment and have not achieved pathological complete remission after surgery, the current standard treatment regimen is 14 cycles of T-DM1 treatment.

In all clinical trials, T-DM1 was found to significantly improve the survival time of these patients and received full approval from the FDA on February, 2013. T-DM1 is now the standard second-line drug for advanced or metastatic breast cancer in many countries, and is currently one of the most successful ADCs.

\section{Trastuzumab deruxtecan}

DS-8201 (also known as "T-DXd") is a new-generation $\mathrm{ADC}$ and was the second ADC to receive approval for advanced HER2-positive breast cancer from the FDA after T-DM1. The differences between DS-8201a and T-DM1 include the linker, cytotoxic drug, and drug:antibody ratio (DAR). The linker in D-S8201 can be cleaved by lysosomes, and the cytotoxic drug in DS-8201 is a topoisomerase inhibitor (derivative of irinotecan). The topoisomerase inhibitor carried by DS-8201 has high potency and can effectively avoid drug resistance. The linker in DS-8201 allows its payload to be released preferentially, which not only ensures the stability in the circulation, but also a tumor-specific killing effect in cells. The DAR of DS-8201a is 8 , which is much higher than the 3.5 of T-DM1 (17).

The ORR of the first-in-human phase I clinical study was $59.5 \%$ in the 111 advanced breast cancer patients. DS-8201 was approved by the FDA in December 2019 and can treat patients with trastuzumab and T-DM1 drug resistance (18). The approval of DS-8201 was based on data from the DESTINY-Breast01 phase II multicenter study that included 184 advanced HER2-positive patients. In the study, DS-8201 significantly benefited these heavily pretreated patients. The ORR was $60.9 \%$, the median PFS was 19.4 months, and the median OS was 20.8 months $(19,20)$. The PFS of brain metastases patients was 18.1 months, indicating that DS-8201 has a significant effect on the treatment of brain metastases. The most noticeable serious grade 3 or higher treatment-related adverse events (TRAEs) were interstitial lung disease.

It is worth noting that DS-8201a also exhibited promising antitumor activity in low HER2 expression breast cancer, with an ORR of $37 \%$ and a median PFS of 11.1 months (21). The details of T-DM1 and DS-8201a are shown in Table 1.

\section{HER2-targeted ADCs currently being investigated}

There is a number of HER2-targeted ADCs are under investigation. The linkers and payloads of these ADCs are different, even the antibody. Here we introduce 7 promising $\mathrm{ADCs}$ at this stage.

\section{TAA013}

TAA013 is formed by connecting trastuzumab and the novel derivative of microtubule inhibitor maytansine (DM1) via a stable thioether bond (trastuzumab-MCC-DM1). TAA013 can be regarded as a similar drug to T-DM1. According to the results announced at the 2020 San Antonio Breast Cancer Symposium (SABCS), the TAA013 phase I study 
has followed the traditional $3+3$ design. In the 22 enrolled recurrent HER2-positive breast cancer patients, TAA013 has been found to be tolerable (22). The phase III study of TAA013 is in progress. Among the HER2-targeted ADCs, the research progress in TAA013 is one of the fastest, and the results are highly anticipated.

\section{Trastuzumab duocarmazine}

Trastuzumab duocarmazine (SYD985) is composed of trastuzumab and synthetic duocarmycin combined with a cleavable vc-linker. The difference from other ADCs is that the payload of this ADC is membrane permeable, meaning it can enter the neighboring cells and cause irreversible DNA damage and cell death through the bystander effect (23). The design used makes it more efficient in low HER2 expression breast cancer but also possibly more toxic. In the related phase I study of 33 HER2-positive local advanced or metastatic breast cancer patients, the ORR was $33 \%$ and the recommended phase II dose (RP2D) was $1.2 \mathrm{mg} / \mathrm{kg}$ (24). The extended study enrolled 146 patients according to the RP2D, including 99 metastatic breast cancer patients who had received multiline therapy, $50 \%$ of whom were HER2 positive. Preliminary results showed that SYD985 had a certain curative effect and that adverse events could be controlled. The TULIP study is an ongoing randomized phase III trial comparing the efficacy of SYD985 and the 3rd-line treatment regimen selected by the investigator in patients with HER2-positive locally advanced or metastatic breast cancer. It is expected that the results of the study will be announced soon.

\section{Vidicuzumab}

Vidicuzumab (RC48-ADC) is a novel HER2-targeted ADC with a novel antibody. The conjugated cytotoxic drug is MMAE, and the linker is a cleavable linker (25). RC48ADC was approved by Chinese National Medicine Products Administration (NMPA) for advanced gastric cancer. As for breast cancer, clinical trials are in progress. In a phase I study including 22 patients with solid tumor, the ORR was $33.3 \%$ (26). The most common TRAEs were bone marrow suppression, alanine transaminase (AST)/aminotransferase (ALT) increase, and peripheral nerve damage. In a phase Ib study including 30 advanced or metastatic breast cancer patients, the ORR for a dose of $2.0 \mathrm{mg} / \mathrm{kg}$ was $46.7 \%$. The phase II and phase III studies are in progress. In addition, another cohort study, C003CANCER, is also exploring the efficacy of RC48-ADC for patients with low HER2 expression, but no data have been disclosed yet.

\section{ARX788}

ARX788, a precisely designed ADC, consists of 2 cytotoxins specific linked to trastuzumab functioning as the basic skeleton. The cytotoxin of ARX788 is AS269. It is characterized by strong toxicity, with a DAR of only 1.8 . According to whether the toxic drug is released through the antibody, it can be classified as a shearable or nonshearable type. The linkers of ARX788 and T-DM1 are both noncleavable, and the antibody, linker, and drug must been cut together intracellularly (27). The advantage of noncutting is that it has a killing effect on breast cancer cells with low HER2 expression and the disadvantage is that it also has a killing effect on normal cells. Therefore, it is necessary to balance the efficacy and toxicity during application. The result of its phase I study that included 45 metastatic breast cancer patients showed ARX788 to be well tolerated and efficacious. The ORR was $56 \%$ with a $1.3-\mathrm{mg} / \mathrm{kg}$ dose cohort and $65 \%$ with a $1.5-\mathrm{mg} / \mathrm{kg}$ dose (27). Bases on these data, the curative effect of ARX788 is similar to that of DS-8201a, and the US FDA has granted it fast-track qualification as a single drug for HER2-positive metastatic breast cancer treatment with 1 or more HER2-targeted regimens. Follow-up clinical trials are underway.

\section{BAT8001}

BAT8001 is formed by covalently connecting trastuzumab and a self-developed linker (6-maleimidohexanoic acid)toxin (maytansine) complex. The results of previous studies have shown that BAT 8001 has a significant inhibitory effect on the proliferation of tumors with high HER2 expression. In the phase I study of BAT8001, the primary endpoint was safety and tolerability of BAT8001, and the secondary endpoint was antitumor activity. The ORR of this study was $38 \%$. Based on these results, BAT8001 was able to forego the phase II clinical trial and enter the phase III clinical trial. In February 2021, the develop company officially announced the preliminary statistical analysis of the BAT 8001 phase III clinical trial. The main efficacy index of PFS in the BAT8001 treatment group was compared with that in the control group (lapatinib combined with capecitabine) but did not achieve the preset superiority target, and the results were unsatisfactory. 
Table 2 HER2-targeted ADCs under investigation for breast cancer

\begin{tabular}{|c|c|c|c|}
\hline ADC & Cytotoxic compound & Linker & Phase \\
\hline SYD985 & Seco-DUBA & Cleavable vc-linker & III \\
\hline RC48-ADC & Monomethyl auristatin E & Cleavable vc-linker & II \\
\hline ARX788 & AS269 & Uncleavable linker & II/III \\
\hline A166 & Monomethylauristatin F & Cleavable vc-linker & II \\
\hline MRG002 & Monomethyl auristatin E & Cleavable vc-linker & II/III \\
\hline
\end{tabular}

HER2, human epidermal growth factor receptor 2; ADC, antibody-drug conjugate.

\section{$A 166$}

A166 is a HER2-targeted ADC with monomethyl auristatin F (MMAF) as its payload. The phase I study yielded a disease control rate (DCR) of $59 \%$ in 27 patients, and the trial is ongoing (28). It should be noted that dry eye and vision blurring are among the most common TRAEs of those treated with A166, which differs from those of other ADCs due to its payload.

\section{MRG002}

MRG002 is a new HER2-targeted ADC, with the monoclonal antibody in the ADC being a sugar-modified trastuzumab and the conjugated cytotoxic drug being MMAE with a cleavable vc-linker. The DAR of MRG002 is 3.6. Sugar-modified trastuzumab can reduce the ability of MRG002 binding to CD16a while ensuring the binding affinity and specificity of the HER2 antigen, thus minimizing the potential killing of immune cells. The conjugated MMAE payload enhances the potential of MRG002 to extend its indications to all cancer types with high expression of HER2, while the safety risks are well controlled, especially the Kadcyla-resistant tumors of MRG002, as indicated in preclinical studies. The cleavable vc-linker helps to release the active MMAE molecules quickly, leading to the death of tumor cells. MRG002 is a promising ADC drug in the tumor treatment field, with its design conferring improvements in safety and efficacy (29).

At the 2021 Chinese Society of Clinical Oncology (CSCO) conference, the results of MRG002 phase I and phase II studies were announced. In the phase I clinical study, a total of 76 patients with HER2-positive advanced solid tumors were enrolled. In dose escalation stage (phase Ia), the recommended dose (RP2D) was determined to be $2.6 \mathrm{mg} / \mathrm{kg}$. In the dose expansion stage (phase Ib), 51 breast cancer patients were enrolled. In terms of safety, the common adverse reactions were mostly at a grade 1 to 2 severity and included decreased neutrophil count (46\%) and increased lactate dehydrogenase (LDH; $43 \%$ ); common (incidence $\geq 10 \%$ ) grade 3 adverse reactions only included a reduction in neutrophil count (17\%); there were no lifethreatening adverse events. Over all, MRG002 is very safe, with controllable adverse reactions that resolve within a short period of time.

In the phase II study of low HER2 expression breast cancer patients, MRG002 has also shown a relatively ideal initial effect. The primary endpoint of the phase II study was ORR, and the secondary endpoints were PFS, duration of response (DOR), DCR, OS and safety. As of September 15,2021 , in terms of efficacy, 5 of the 18 patients that could be evaluated have reached partial response (PR) for the first efficacy evaluation. In terms of safety, the performance of this study is similar to that of the phase I study, with low toxicity and easy management. Another MRG002 multicenter phase II study in the treatment of HER2 positive advanced breast cancer is also in progress.

Based on the above 2 studies, MRG002 has initially demonstrated its efficacy in both patients with both low and high HER2 expression. It is also hoped that the phase III clinical study will be conducted soon after the phase II clinical study is completed so a greater variety and quality of clinical options can be provided to patients as soon as possible.

The details of the ADCs described above are shown in Table 2. 


\section{Other HER2-targeted ADCs under investigation}

MM302, another HER2-targeted ADC, has pegylated antibody-liposomal doxorubicin as its payload. Although the phase I result is promising (30), the phase II study proved to be a failure (31). Another HER2-targeted ADC, XMT-1522, has MMAF as its payload. The company developing this drug needed to halt the research due to the fierce competitive environment of HER2-targeted ADCs, although the phase I result had shown some promising data.

\section{Discussion}

HER2-targeted ADCs have been able to appreciably extend the survival of HER2-positive patients and provide a satisfactory effect in breast cancer patients with low HER2 expression. However, some questions remain to be answered. For instance, some ADCs have even greater serious adverse reactions than do chemotherapeutic drugs, and some release cytotoxic drugs into the blood before their monoclonal antibodies partially bind to cancer cell receptors. With the progress of related technology, these problems will likely be solved in time.

With the popularization of HER2-targeted ADCs applications, drug resistance will inevitably arise. Similar to when the use of programmed cell death protein 1/programmed death-ligand 1 (PD-1/PD-L1) antibodies first emerged, cancer patients saw a new hope. However, after a few months of treatment, many patients develop drug resistance. This problem has not yet been resolved, and ADC therapy will also face similar challenges. Combination with other drugs, such as PD-1/PD-L1, may be a promising alternative, and researchers are already conducting research toward this end, with the preliminary research results being satisfactory.

Exploring the effective biomarkers for predicting the prognosis of ADCs and searching for potential ADCs in breast cancer patients are also important future research directions for refining the clinical application of ADCs.

\section{Conclusions}

The approved HER2-targeted ADCs have significantly benefited breast cancer patients, and the ones being currently investigated may provide even further benefit. The combination of ADC with other drugs may also prove valuable in treating patients with HER2-positive breast cancer and those with low HER2 expression.

\section{Acknowledgments}

Funding: None.

\section{Footnote}

Reporting Checklist: The authors have completed the Narrative Review reporting checklist. Available at https:// dx.doi.org/10.21037/tbcr-21-33

Conflicts of Interest: All authors have completed the ICMJE uniform disclosure form (available at https://dx.doi. org/10.21037/tbcr-21-33). The authors have no conflicts of interest to declare.

Ethical Statement: The authors are accountable for all aspects of the work in ensuring that questions related to the accuracy or integrity of any part of the work are appropriately investigated and resolved.

Open Access Statement: This is an Open Access article distributed in accordance with the Creative Commons Attribution-NonCommercial-NoDerivs 4.0 International License (CC BY-NC-ND 4.0), which permits the noncommercial replication and distribution of the article with the strict proviso that no changes or edits are made and the original work is properly cited (including links to both the formal publication through the relevant DOI and the license). See: https://creativecommons.org/licenses/by-nc-nd/4.0/.

\section{References}

1. Miller DR. A tribute to Sidney Farber-- the father of modern chemotherapy. Br J Haematol 2006;134:20-6.

2. Köhler G, Milstein C. Continuous cultures of fused cells secreting antibody of predefined specificity. Nature 1975;256:495-7.

3. Lambert JM, Morris CQ. Antibody-Drug Conjugates (ADCs) for Personalized Treatment of Solid Tumors: A Review. Adv Ther 2017;34:1015-35.

4. Lambert JM, Berkenblit A. Antibody-Drug Conjugates for Cancer Treatment. Annu Rev Med 2018;69:191-207.

5. Juan A, Cimas FJ, Bravo I, et al. An Overview of Antibody Conjugated Polymeric Nanoparticles for Breast Cancer Therapy. Pharmaceutics 2020;12:802.

6. Juan A, Cimas FJ, Bravo I, et al. Antibody Conjugation of Nanoparticles as Therapeutics for Breast Cancer Treatment. Int J Mol Sci 2020;21:6018. 
7. Ferraro E, Drago JZ, Modi S. Implementing antibodydrug conjugates (ADCs) in HER2-positive breast cancer: state of the art and future directions. Breast Cancer Res 2021;23:84.

8. Lambert JM, Chari RV. Ado-trastuzumab Emtansine (TDM1): an antibody-drug conjugate (ADC) for HER2positive breast cancer. J Med Chem 2014;57:6949-64.

9. Corrigan PA, Cicci TA, Auten JJ, et al. Ado-trastuzumab emtansine: a HER2-positive targeted antibody-drug conjugate. Ann Pharmacother 2014;48:1484-93.

10. Krop IE, Beeram M, Modi S, et al. Phase I study of trastuzumab-DM1, an HER2 antibody-drug conjugate, given every 3 weeks to patients with HER2-positive metastatic breast cancer. J Clin Oncol 2010;28:2698-704.

11. Baselga J. Phase I and II clinical trials of trastuzumab. Ann Oncol 2001;12 Suppl 1:S49-55.

12. Beeram M, Krop IE, Burris HA, et al. A phase 1 study of weekly dosing of trastuzumab emtansine (T-DM1) in patients with advanced human epidermal growth factor 2-positive breast cancer. Cancer 2012;118:5733-40.

13. Burris HA 3rd, Rugo HS, Vukelja SJ, et al. Phase II study of the antibody drug conjugate trastuzumab-DM1 for the treatment of human epidermal growth factor receptor 2 (HER2)-positive breast cancer after prior HER2-directed therapy. J Clin Oncol 2011;29:398-405.

14. Krop IE, LoRusso P, Miller KD, et al. A phase II study of trastuzumab emtansine in patients with human epidermal growth factor receptor 2-positive metastatic breast cancer who were previously treated with trastuzumab, lapatinib, an anthracycline, a taxane, and capecitabine. J Clin Oncol 2012;30:3234-41.

15. Welslau M, Diéras V, Sohn JH, et al. Patient-reported outcomes from EMILIA, a randomized phase 3 study of trastuzumab emtansine (T-DM1) versus capecitabine and lapatinib in human epidermal growth factor receptor 2 -positive locally advanced or metastatic breast cancer. Cancer 2014;120:642-51.

16. Krop IE, Kim SB, González-Martín A, et al. Trastuzumab emtansine versus treatment of physician's choice for pretreated HER2-positive advanced breast cancer (TH3RESA): a randomised, open-label, phase 3 trial. Lancet Oncol 2014;15:689-99.

17. Perez J, Garrigós L, Gion M, et al. Trastuzumab deruxtecan in HER2-positive metastatic breast cancer and beyond. Expert Opin Biol Ther 2021;21:811-24.

18. Narayan P, Osgood CL, Singh H, et al. FDA Approval Summary: Fam-Trastuzumab Deruxtecan-Nxki for the Treatment of Unresectable or Metastatic HER2-Positive
Breast Cancer. Clin Cancer Res 2021;27:4478-85.

19. Modi S, Saura C, Yamashita T, et al. Trastuzumab Deruxtecan in Previously Treated HER2-Positive Breast Cancer. N Engl J Med 2020;382:610-21.

20. Modi S, Saura C, Yamashita T, et al. Abstract PD306: Updated results from DESTINY-breast01, a phase 2 trial of trastuzumab deruxtecan (T-DXd) in HER2 positive metastatic breast cancer. Cancer Res 2021. doi: 10.1158/1538-7445.SABCS20-PD3-06.

21. Modi S, Park H, Murthy RK, et al. Antitumor Activity and Safety of Trastuzumab Deruxtecan in Patients With HER2-Low-Expressing Advanced Breast Cancer: Results From a Phase Ib Study. J Clin Oncol 2020;38:1887-96.

22. Liu JM, Yin YM, Wu H, et al. Abstract PS10-51: TAA013 a trastuzumab antibody drug conjugate phase I dose escalation study in recurrent her2 positive breast cancer. Cancer Res 2021. doi: 10.1158/1538-7445.SABCS20PS10-51

23. Dokter W, Ubink R, van der Lee $M$, et al. Preclinical profile of the HER2-targeting ADC SYD983/SYD985: introduction of a new duocarmycin-based linker-drug platform. Mol Cancer Ther 2014;13:2618-29.

24. Banerji U, van Herpen CML, Saura C, et al. Trastuzumab duocarmazine in locally advanced and metastatic solid tumours and HER2-expressing breast cancer: a phase 1 dose-escalation and dose-expansion study. Lancet Oncol 2019;20:1124-35

25. Li L, Xu MZ, Wang L, et al. Conjugating MMAE to a novel anti-HER2 antibody for selective targeted delivery. Eur Rev Med Pharmacol Sci 2020;24:12929-37.

26. Gong J, Shen L, Wang W, et al. Safety, pharmacokinetics and efficacy of RC48-ADC in a phase I study in patients with HER2-overexpression advanced solid cancer. J Clin Oncol 2018;36:e16059.

27. Skidmore L, Sakamuri S, Knudsen NA, et al. ARX788, a Site-specific Anti-HER2 Antibody-Drug Conjugate, Demonstrates Potent and Selective Activity in HER2-low and T-DM1-resistant Breast and Gastric Cancers. Mol Cancer Ther 2020;19:1833-43.

28. Liu Y, Lian W, Zhao X, et al. A first in-human study of A166 in patients with locally advanced/metastatic solid tumors which are HER2-positive or HER2-amplified who did not respond or stopped responding to approved therapies. J Clin Oncol 2020;38:abstr 1049.

29. Li H, Zhang X, Xu Z, et al. Preclinical evaluation of MRG002, a novel HER2-targeting antibody-drug conjugate with potent antitumor activity against HER2positive solid tumors. Antib Ther 2021;4:175-84. 
30. Munster P, Krop IE, LoRusso P, et al. Safety and pharmacokinetics of MM-302, a HER2-targeted antibodyliposomal doxorubicin conjugate, in patients with advanced HER2-positive breast cancer: a phase 1 dose-escalation study. Br J Cancer 2018;119:1086-93.

31. Miller K, Cortes J, Hurvitz SA, et al. HERMIONE: a

doi: $10.21037 /$ tbcr-21-33

Cite this article as: Li Q, Guo Y, Lin F, Li L, Ge X, Zhao W, Li J. A narrative review of human epidermal growth factor receptor 2 (HER2)-targeted antibody-drug conjugates in the treatment of breast cancer. Transl Breast Cancer Res 2021;2:27. randomized Phase 2 trial of MM-302 plus trastuzumab versus chemotherapy of physician's choice plus trastuzumab in patients with previously treated, anthracycline-naïve, HER2-positive, locally advanced/metastatic breast cancer. BMC Cancer 2016;16:352. 